\title{
Estimación de máximos niveles de sismicidad para el Litoral Ecuatoriano a través de la integración de datos geológicos y sismotectónicos
}

\author{
Kervin CHUNGA ${ }^{(1)}$, Carlos MARTILLO ${ }^{(2)}$, Nelson PAZMIÑO ${ }^{(3)}$, \\ Ma. Fernanda QUIÑÓNEZ ${ }^{(3)}$, Freddy HUAMAN ${ }^{(1)}$
}

(1) Centro de Investigaciones de Geociencias CIGEO. Facultad de Ciencias de la Ingeniería. Universidad Estatal Península de Santa Elena UPSE, La Libertad, Ecuador.

(2) ESPOL, Facultad de Ingeniería en Ciencias de la Tierra. Campus Gustavo Galindo, La Prosperina, Guayaquil, Ecuador.

(3) Instituto Oceanográfico de la Armada - INOCAR. Base Naval Sur, Avda. 25 de Julio. Vía Pto. Marítimo. P.O.Box.5940. Guayaquil-Ecuador.

\begin{abstract}
Resumen
Una evaluación de riesgo sísmico para el litoral ecuatoriano es presentada en este artículo. La máxima magnitud estimada y el máximo desplazamiento lateral para cada falla activa y capaz es calculada usando la relación empirica de regresión de magnitud-ruptura/desplazamiento de falla, propuesta por Well \& Coppersmith (1994). La distribución espacial de los sismos corticales y modelos tectónicos de subducción han permitido individualizar posibles estructuras sismogenéticas capaces de generar terremotos con magnitudes mayor a 6. Para el territorio continental del Ecuador, han sido seleccionado 129 terremotos históricos (escalas de intensidades macrosísmicas MSK-1956 y ESI-2007), 985 grados de intensidades correspondientes a reportes de daños en el terreno y viviendas (fuente: IG-EPN) y 5997 datos de sismicidad instrumental (Catálogos seleccionados NEIC, CERESIS y IG-EPN) entre 4,0 a 8,8. Los fenómenos geológicos cosísmicos que podrían generarse durante un terremoto en la ciudad de Guayaquil fueron también individualizados en este estudio.
\end{abstract}

Palabras claves: Intensidad macrosísmica, modelo tectónico de subducción, Litoral de Ecuador.

\begin{abstract}
A seismic risk assessment study relative to the Ecuadorian coasts is presented in this report. The likely maximum magnitude and the maximum offset for each active and capable fault are calculated using empiric regression law between magnitude and offset/rupture (Well \& Coppersmith, 1994). Seismic spatial distribution and subduction tectonic models allowed identifying seismogenic structures in the crustal and megathrust earthquake areas. Based on 129 historical earthquakes, 985 maximum felt intensity data (MSK-1956 and ESI2007 scales) and 5997 instrumental seismicity data among $M w$ 4,0 and 8,8, they seismicity level for the Ecuadorians coasts has been evaluated. Secondary geological effects that could be generated during an crustal earthquake in the Guayaquil city area were also hypotized in this study.
\end{abstract}

Keywords: Macroseismic Intensity, Subduction tectonic Models, Ecuadorian Coastal.

\section{Introducción}

El análisis de la sismicidad histórica e instrumental del litoral ecuatoriano fue realizado usando información de tres catálogos sísmicos: (1) Centro Regional de sismología para América del Sur - CERESIS, (2) National Earthquake Information Center NEIC, e (3) Instituto Geofísico - Escuela Politécnica Nacional del Ecuador, IGEPN. La complementación de todos los datos hipocentrales y la distribución espacial de los mismos, permitió individualizar las zonas tectónicas corticales (también definidas como superficiales) asociadas a fallas geológicas con distancia hipocentral $\leq 40 \mathrm{Km}$ de profundidad. La fosa tectónica representa la colisión y subducción de la placa oceánica de Nazca contra el segmento continental conformado por el Bloque Norandino y la placa Sudamericana, donde terremotos con magnitudes mayor a 7 pueden ocurrir. 
El Litoral ecuatoriano es caracterizado por una complejidad morfo-estructural, en la parte sur, en el Golfo de Guayaquil sistemas distensivos son asociadas a la abertura de la corteza continental a través de fallamientos de tipo normales y de cizallas, un rasgo distinto es presente en el prisma de acreción junto al límite de la fosa tectónica de subducción, donde sistemas de fallas inversas de bajo ángulo de cerca de $70 \mathrm{Km}$ de longitud podrían indicar una estructura sismogénica "fallas megasplay" (ie. Chunga \& Quiñónez, 2013) capaz de generar sismos mayor a magnitud 7 y también desplazamientos verticales con generación de ondas anómalas de tsunamis, sin descartar potenciales deslizamientos submarinos. En la parte central, terrazas marinas plio-cuaternarias evidencian un levantamiento tectónico con moderadas a altas tasas de desplazamientos (expresada en $\mathrm{mm} / \mathrm{año}$ ), esta dinámica de deformación cortical se debe al empuje de la cordillera oceánica de Carnegie y posterior subducción contra el segmento continental, en la parte norte, sismos delineados en el sector Galera (sur de la provincia de Esmeraldas) indican el inicio de la cuenca de subsidencia de Esmeraldas y de la cuenca de Borbón las cuales son delimitadas por el levantamiento horst de Viche. Todos estos rasgos morfo-estructurales y contrastes con los relieves y cuencas de ante-arcos son atribuidos a la tectónica activa interplaca.

En las siguientes secciones de este documento, se analizará la información sismológica con distancia hipocentral entre los 1 y $40 \mathrm{Km}$ de profundidad, así como relación con delineadas fallas geológicas activas. Desde estas perspectivas sismotectónicas, y estimando la máxima magnitud para cada segmento de falla se elaboran modelos de isosistas de intensidades macrosísmicas para el litoral Ecuatoriano.
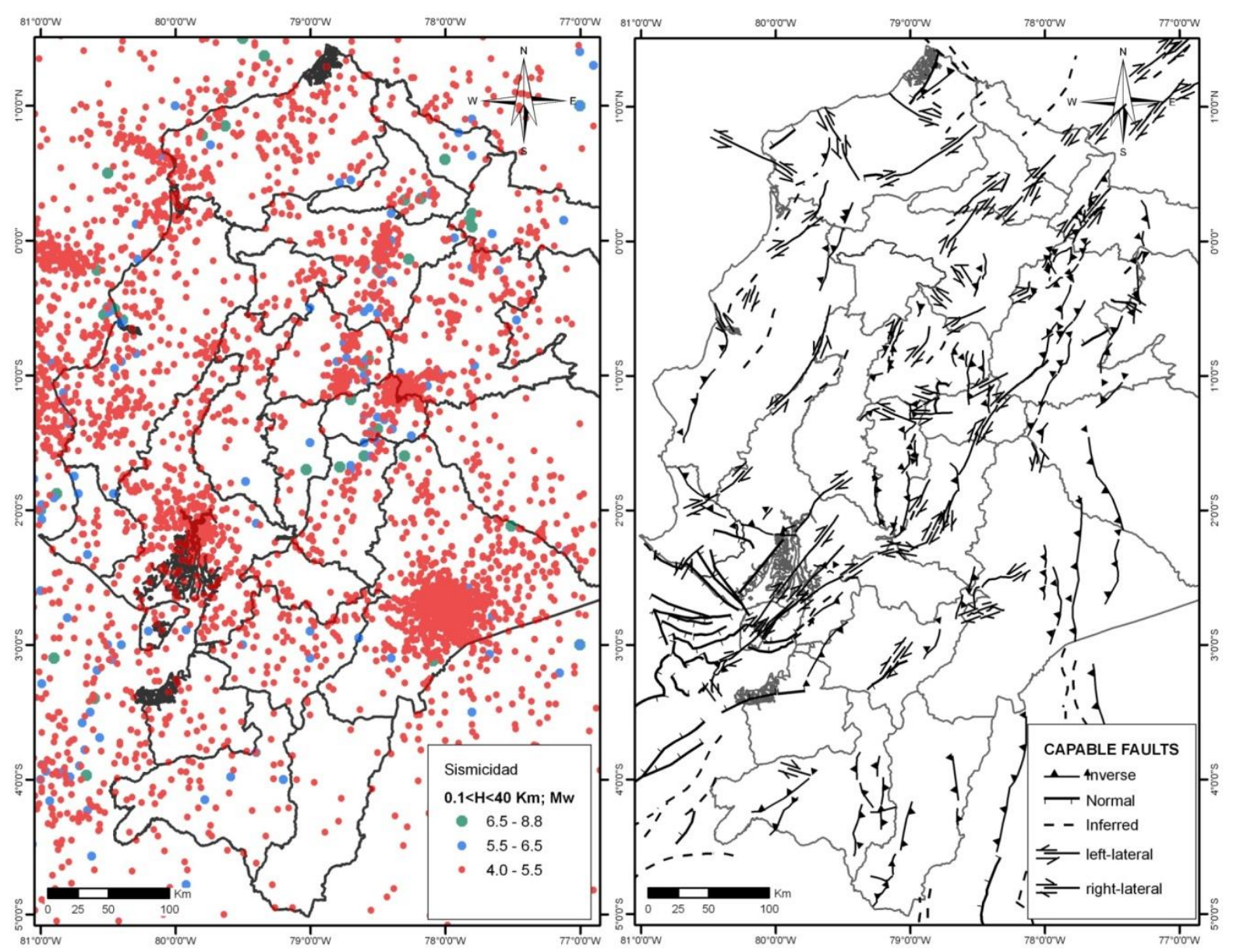

Figura 1. Sismicidad y ambiente tectónico en Ecuador. Izquierda, distribución espacial de sismos corticales instrumentales con $4 \leq \mathrm{M} \leq 8.8$. Derecha, cinemática y tipo de fallas capaces cartografiada para el territorio del Ecuador y regiones fronterizas de Colombia y Perú (Chunga et al., 2011). 


\section{Sismotectónica}

Tectónicamente, el área seleccionada en estudio está dentro de una zona interplaca, el entorno geodinámico de la fosa Ecuador es complejo y tiene su origen en la convergencia de la placas oceánica (Nazca) y Continental (Sudamericana) dentro del proceso de subducción (Beck \& Susan, 1992; Eguez et al., 2003; Espinoza, 1992; Mendoza \&
Dewey, 1984). Está convergencia de placas es también conocida como "zona de subducción" o "borde occidental de Sudamérica" (Figura 1), y ha dado origen a diferentes niveles de deformación en la corteza continental hasta la formación de cadenas de altas colinas (máxima altitud 760 m.s.n.m) y zonas de subsidencia tectónica en la región costera del Ecuador.

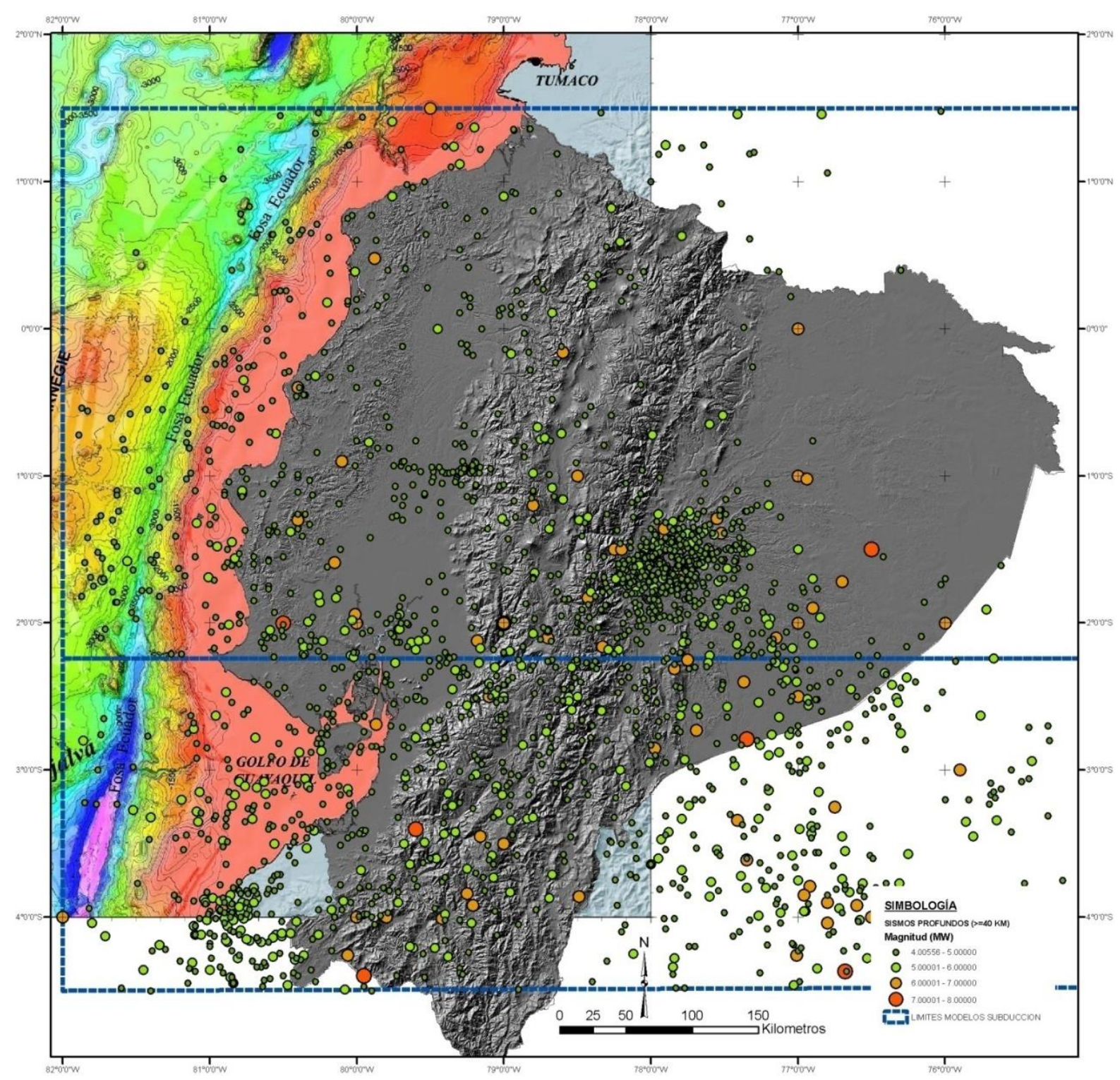

Figura 2. Distribución espacial de sismos superficiales y profundos en el Ecuador continental y parte del segmento oceánico. Revisando los lineamientos estructurales propuestos por Chunga et al. (2011), se ha delineado el territorio en dos áreas distintas (zona norte y sur, respectivamente), la proyección de los sismos hipocentrales son observados en la Figura 3. 
Está región tectónica ecuatoriana es conocida también como "zona de subducción" o "fosa oceánica sudamericana", donde los registros sísmicos ecuatorianos reportan fuertes terremotos de $\mathrm{Mw}>$ 7.75; el sismo de mayor magnitud Ms 8.8 (otros catálogos reportan $\mathrm{Mw}$ 8.7, Mt 8.7) fue generado el 31 de enero de 1906, a una profundidad de $25 \mathrm{Km}$, costa afuera $138 \mathrm{Km} \mathrm{W}$ del sitio Tortuga, Esmeraldas, causando entre 500 a 1500 víctimas mortales (Elizalde, 1985; Gutscher et al., 1999). Este es el sexto terremoto más fuerte en el mundo registrado en los últimos 110 años. Gustcher et al. (1999), atribuye el origen del terremoto de
1906 a un desplazamiento de ruptura cortical de cerca $500 \mathrm{Km}$ de longitud, que se extiende costa afuera desde los límites de Bahía de Caráquez (Manabí, Ecuador) hasta Tumaco (Colombia); posteriormente ha sido reactivada en 1942 (M 7.9), 1958 (M 7.8) y 1979 (M 8.1). El terremoto de 1998 en Bahía de Caráquez (M 7.1), también puede ser considerado como consecuencia de la reactivación de la mega-ruptura anteriormente mencionada. En la fosa ecuatoriana - Golfo de Guayaquil - norte de Perú, se han registrado seis terremotos $6,9 \leq \mathrm{M} \leq 7,8$, en los años 1901, 1933, 1953, 1959 (Silgado, 1957; Swenson \& Beck, 1996). (Ver Tabla 1).

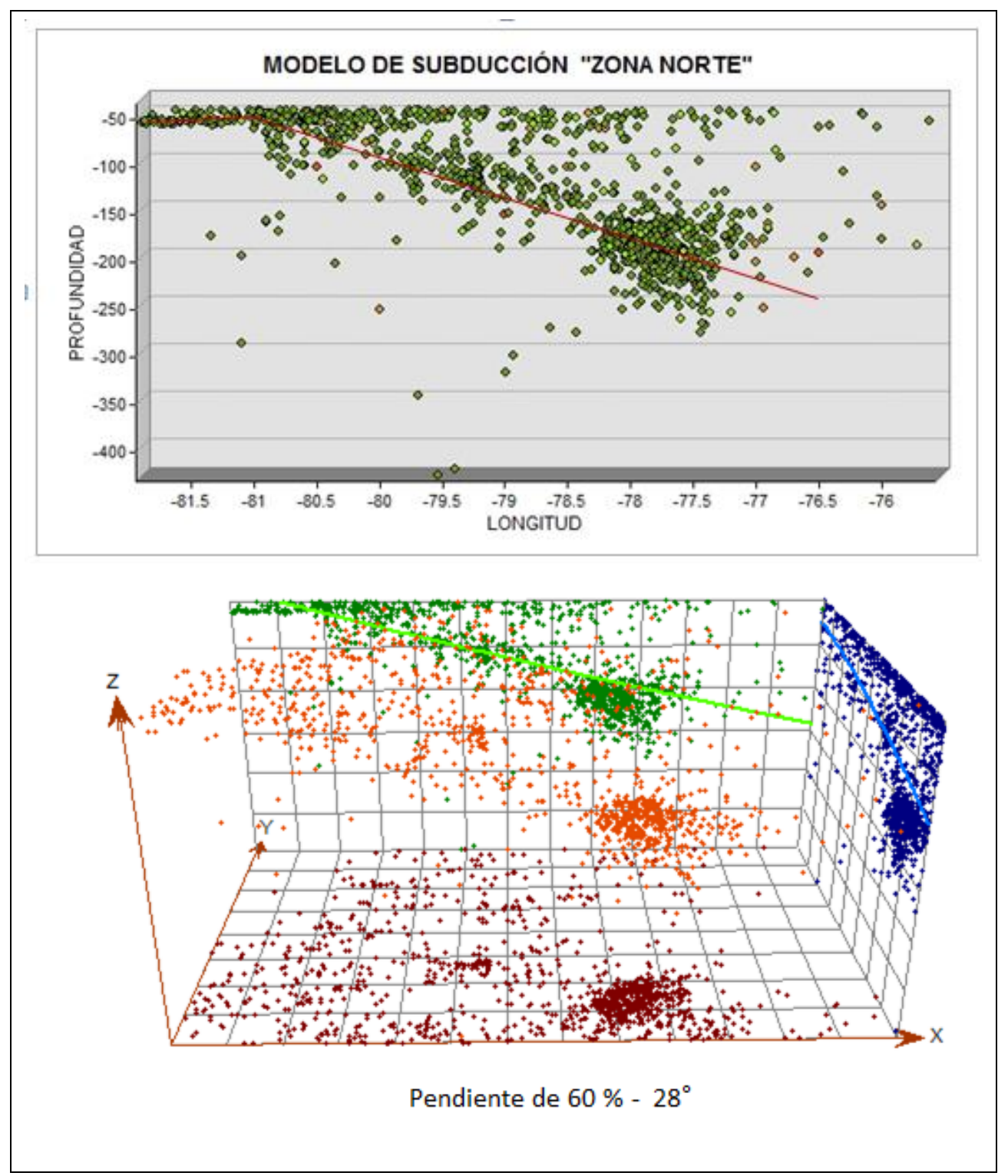

Figura 3. Modelo de Subducción Zona Norte, con un ángulo de subducción del "slab" de $28^{\circ}$. Imagen superior representa la proyección de sismos tectónicos en dos dimensiones. En la imagen inferior se observa la proyección espacial (3D) de sismos tectónicos. 
Si confrontamos esta realidad tectónica con una de las zonas de convergencia más activas en el mundo, la placa tectónica Indiana y la placa Birmania (responsables del terremoto de Sumatra del 24 de diciembre de 2004, Mw 9.3, hipocentro $30 \mathrm{Km})$; en donde, la tasa de tendencia o desplazamiento de la placa Indiana es de 60-75 $\mathrm{mm} / \mathrm{año}$, dirección $\mathrm{N} 3^{\circ}-\mathrm{N} 25^{\circ}$. Y con dos de las más rápidas fallas laterales dextrales en el mundo, la falla gran Sumatra y Mentawai, (según, Bellier et al., 1997) que tiene una tasa estimada de desplazamiento lateral de 40 a $60 \mathrm{~mm} / \mathrm{año} \mathrm{(Petersen}$ et al., 2004). La similitud tectónica para Ecuador en las tasas de tendencias para la zona de subducción de las placas de Nazca y la Sudamericana también la convierten, por lo tanto, en una de las más activas del mundo.

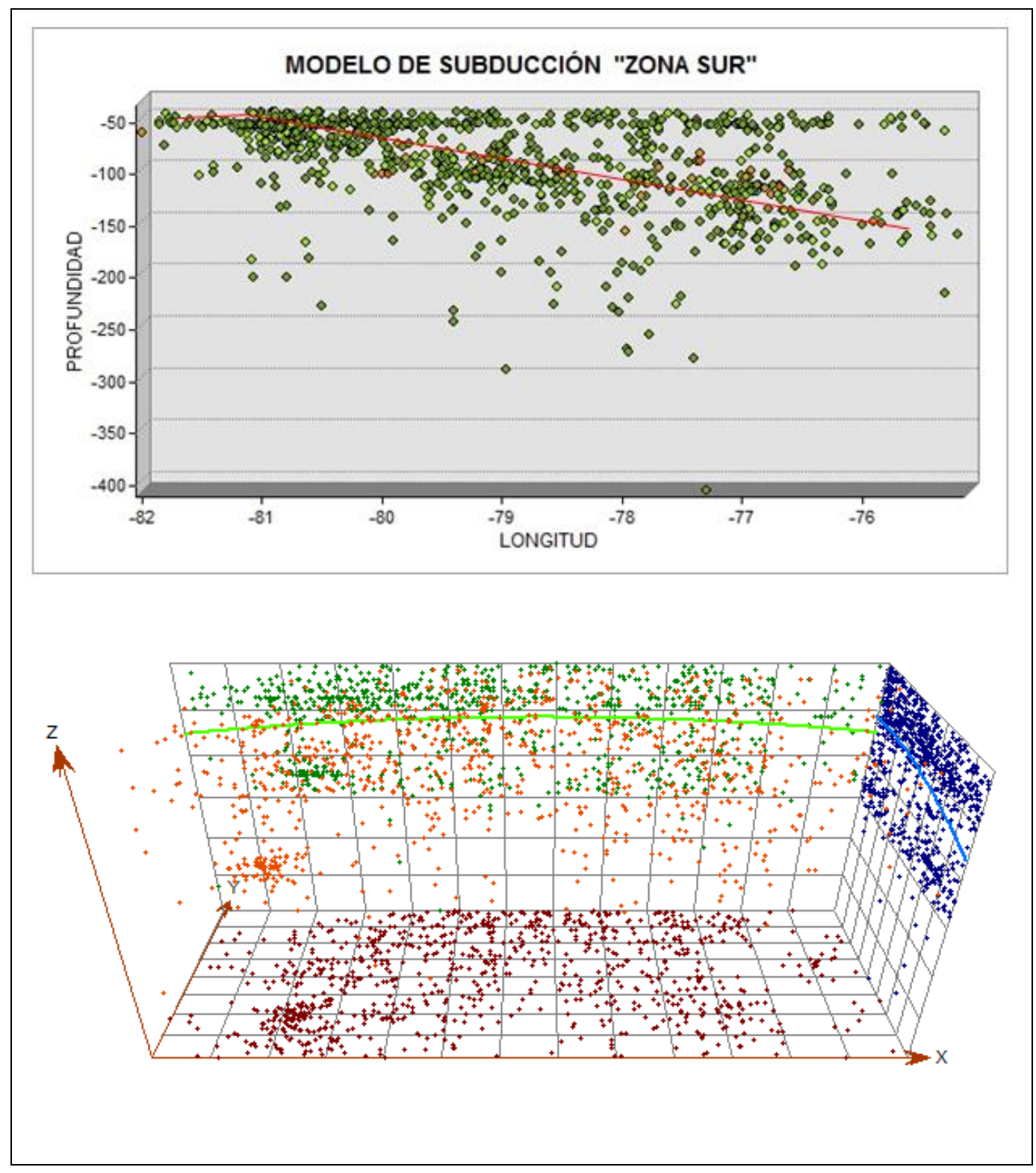

Figura 4. Modelo de Subducción Zona Sur, con un ángulo de subducción del slab de $17^{\circ}$. Imagen superior representa la proyección de sismos tectónicos en dos dimensiones. Imagen inferior indica la proyección espacial (3D) de sismos tectónicos. 
La tasa de desplazamiento de la placa de Nazca es de cerca 6 a $8 \mathrm{~cm}$ por año (Baldock 1983, Barazangi \& Isacks 1976, Bourdon \& Eissen et al. 2003, Dumont et al., 2005; Gustcher et al., 1999, White et al., 2003), parte de ese desplazamiento se transfiere a la deformación continental cortical de la placa Sudamericana y del Bloque Norandino localizado en la zona estructural de abertura del Golfo de Guayaquil. La tasa de desplazamiento del Bloque Norandino es de cerca $8.7 \mathrm{~mm} / \mathrm{a}$ (Nocquet et al., 2010), con dirección $\mathrm{N} 35 \mathrm{E}$ respecto a la Sudamericana a lo largo de la mega falla de cizalla Guayaquil - Caracas (MFGC). Esta mega falla se extiende desde el Golfo de Guayaquil hasta la costa norte de Venezuela. Dumont et al. (2005), Santana et al. (2003), y Winter et al. (1993), indican que el límite SW de la MFGC (área del Golfo de Guayaquil) tiene tasa de deformación lateral destral y sinestral alcanzando entre los 3 a $9 \mathrm{~mm}$ por año, sugiriendo que el resto del total de $10 \mathrm{~mm}$ por año de deformación cortical es acomodado por otros sistemas de fallas de cizallas segmentadas en el Golfo de Guayaquil.

Los eventos superficiales (distancia focal $0.1 \leq \mathrm{H} \leq 40 \mathrm{Km}$ ) representan los terremotos corticales asociados a sistemas de fallas activas y capaces de deformar considerablemente la superficie del terreno (Chunga 2010, Riva et al. 2007, Tassara 2003, White et al. 2003); los eventos profundos $(40 \leq \mathrm{H} \leq 286 \mathrm{Km}$ distancia focal) en la zona de slab inmergen considerablemente a medida que se aleja de la fosa o trinchera Ecuador (Swenson \& Beck, 1996). La zona slab es localizada dentro de la zona tectónica de Benioff-Wadati (Pennington 1981, Vannucchi \& Galeotti 2004, Vargas 2005).

\begin{tabular}{|c|c|c|c|c|c|c|c|c|c|c|}
\hline Año & Mes & Día & Lat. & Long. & Fuente Sismogenética & Ms & Mw & $\begin{array}{c}\text { Foco } \\
\mathrm{Km}\end{array}$ & $\begin{array}{c}\mathrm{H} \\
\mathrm{mt}\end{array}$ & It \\
\hline 1877 & 10 & 11 & 2,30 & $-78,78$ & Fosa Ecua-Col & & & & & \\
\hline 1901 & 1 & 7 & $-2,00$ & $-82,00$ & Fosa Ecua-Perú & 7,6 & 7,0 & 25 & & \\
\hline 1901 & 1 & 8 & $-2,00$ & $-82,00$ & Fosa Ecua-Perú & 7,1 & 6,7 & & & \\
\hline 1906 & 1 & 31 & 1,00 & $-80,00$ & Fosa Ecuador & 5,7 & 5,9 & 25 & & \\
\hline 1906 & 1 & 31 & 1,00 & $-81,50$ & Prisma Acreción & 8,6 & 8,8 & 25 & 5 & 3 \\
\hline 1906 & 2 & 7 & 1,00 & $-81,00$ & Prisma Acreción & & & & & 0.5 \\
\hline 1907 & 6 & 1 & 0,00 & $-82,00$ & Fosa Ecuador & 7,4 & 6,9 & 33 & & \\
\hline 1933 & 10 & 2 & $-2,20$ & $-81,04$ & Fosa Ecuador & 6,9 & 6,6 & 10 & 0.2 & \\
\hline 1942 & 5 & 14 & $-0,70$ & $-81,50$ & Fosa Ecuador & 8,3 & 7,6 & 10 & & \\
\hline 1942 & 7 & 4 & 0,80 & $-80,50$ & Fosa Ecuador & 6,2 & 6,4 & 15 & & \\
\hline 1953 & 12 & 12 & $-3,50$ & $-81,00$ & Fosa Ecua-Perú & 7,3 & 6,8 & 33 & & \\
\hline 1953 & 12 & 12 & $-3,60$ & $-80,60$ & Fosa Ecua-Perú & 7,8 & 7,4 & 30 & 1.8 & 0.5 \\
\hline 1956 & 1 & 16 & $-0,50$ & $-80,50$ & Fosa Ecuador & 7,3 & 6,8 & & & \\
\hline 1958 & 1 & 19 & 1,37 & $-79,34$ & Fosa Ecuador & 7,3 & 7,9 & 20 & 0 & 1.5 \\
\hline 1958 & 8 & 17 & 1 & $-80,00$ & Fosa Ecuador & 6,1 & 6,3 & & & \\
\hline 1959 & 2 & 7 & $-3,84$ & $-81,58$ & Fosa Ecua-Perú & 7,2 & 6,8 & 33 & 0.2 & -2 \\
\hline 1979 & 12 & 12 & 1,60 & $-79,36$ & Fosa Ecua-Col & 7,7 & 7,8 & 24 & 5 & 2.5 \\
\hline 1989 & 6 & 25 & 1,1 & $-79,60$ & Fosa Ecuador & 6,4 & 6,6 & 33 & & \\
\hline 1998 & 8 & 4 & $-0,59$ & $-80,39$ & Fosa Ecuador & 7,1 & 6,7 & 33 & & \\
\hline
\end{tabular}

Tabla 1. Terremotos de $6,9 \leq \mathrm{M} \leq 8,8$ generados en la fosa Ecuador y con distancia focal $\leq 33 \mathrm{Km}$ de profundidad. Ms, magnitud ondas superficiales. Mw, momento de magnitud. Foco, distancia hipocentral. $\mathrm{H}$, altura máxima de ola de tsunami registrada en la línea de costa. It, escala de intensidad de Tsunamis Soloviev-Inamura. Los sismos marcados con blanco han generado tsunamis (Fuente: catálogo sísmico NEIC). 


\section{Modelos de Subducción}

Para comprender la distribución espacial de los sismos y su asociación a mega-estructuras sismogénicas asociadas a la geodinámica de la zona de subducción, hemos elaborado dos modelos de subducción para el territorio del Ecuador. Una investigación similar ha sido efectuada por Gutscher et al. (1999). En la Figura 5, se delimitan en superficie los segmentos Norte y Sur. Para cada segmento modelamos el slab o plano de desplazamiento de la placa de Nazca con respecto al bloque Norandino y la placa Sudamericana. El slab o plano de Benioff en la región costa tiene una inmersión estructural entre 4 a $5^{\circ}$, entre los 40 a 70 $\mathrm{Km}$ de profundidad, un cambio brusco de inmersión es delineado en la región andina alcanzando su máxima inclinación por debajo de la cuenca retroarco amazónica, con plano de inclinación entre los $17^{\circ}$ a $28^{\circ}$.

Las magnitudes máximas para cada segmento se demuestran en la Tabla 2. La longitud de la zona de subducción en el segmento Norte es de $410 \mathrm{Km}$ y del segmento Sur 270 Km (Figura 2).

Los sismos con foco superficial $(0.1 \leq \mathrm{H} \leq 35 \mathrm{Km})$ se presentan con mayor concentración en el Modelo Norte, entre la fosa oceánica y las áreas de costa, siendo estos asociados al proceso de subducción de la placa de Nazca bajo la Sudamericana a profundidades menores a $35 \mathrm{~km}$, y a una elevada tasa de compresión provocada por la colisión de la cordillera de Carnegie (Figura 3). En el interior del continente, también existe presencia de sismos superficiales y estos se distribuyen en los modelos propuestos, en las planicies de las cuencas costeras de arte-arcos, a lo largo de la zona subandina y en las altas cordilleras de los Andes, todas ellas asociadas a las deformaciones superficiales con la presencia de importantes sistemas de fallas corticales activas y capaces.

En el Modelo Norte, la magnitud máxima que puede generar un terremoto esta entre los 7.2 y 8.8, relacionándolo también con la ruptura de cerca de $500 \mathrm{Km}$ considerando su extensión estructural hasta Tumaco, que fue la estructura sismogenética que generó el terremoto de M 8.8 en Esmeraldas, el 31 de enero de 1906; subsecuentes otras reactivaciones fueron reportadas en 1942, 1958 y 1979 (ver Tabla 1). En el segmento del Modelo Sur, la máxima M estimada está entre los M 7,1 a 7.8, el sismo más reciente con efecto local aconteció el 10.12.1970 con M 7.1. Una tasa de desplazamiento de 60 a 79 $\mathrm{mm} / \mathrm{año}$ es asignada a cada segmento basado sobre la tasa de convergencia estimada.

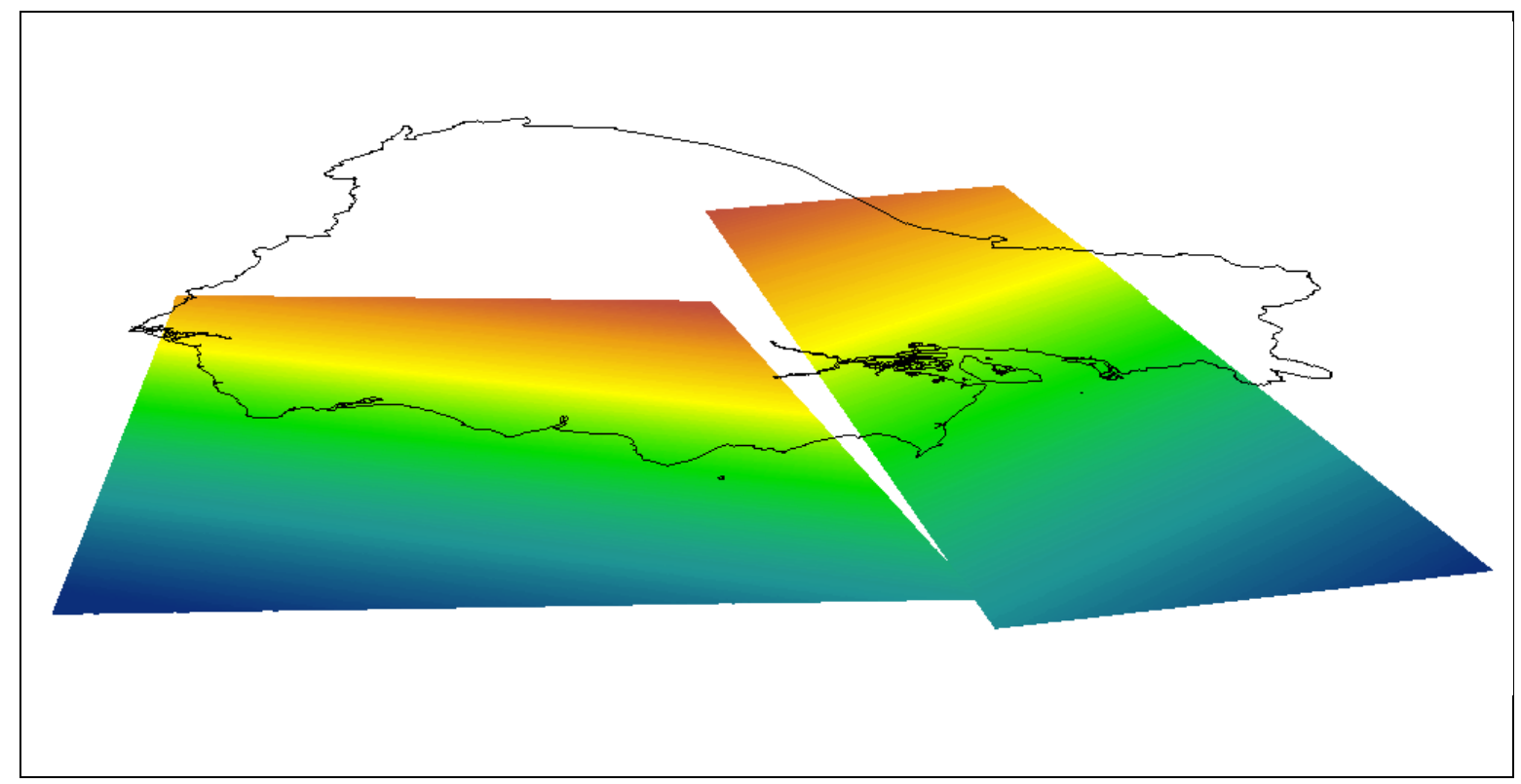

Figura 5. Modelos de los planos de subducción frente al Ecuador. Nótese que el sector Norte $\left(28^{\circ}\right)$ presenta un ángulo mayor de subducción que la Zona Sur (17). 


\begin{tabular}{|c|c|c|c|c|c|c|}
\hline SEG & LAT & LS (Km) & IE & $\begin{array}{l}\text { Magnitud } \\
(\mathrm{Mw})\end{array}$ & Terremotos históricos & $\begin{array}{c}\mathrm{T} \\
\text { (mm/año) }\end{array}$ \\
\hline Norte & $+1.5 ;-2.3$ & 410 & $28^{\circ}$ & 9 & $\begin{array}{l}31.01 .1906 \text { (M 8.8) } \\
02.10 .1942 \text { (M 7.8) } \\
\text { 19.01.1958 (M 7.7) } \\
\text { 12.12.1979 (M 8.2) } \\
04.08 .1998 \text { (M 7.2) }\end{array}$ & \multirow{2}{*}{$68 \pm 10$} \\
\hline Sur & $-2.3 ;-4.5$ & 270 & $17^{\circ}$ & 8.8 & $\begin{array}{l}\text { 07.01.1901 (M 7.8) } \\
12.12 .1953 \text { (M7.8) } \\
07.02 .1959 \text { (M7.4) } \\
10.12 .1970 \text { (M 7.1) }\end{array}$ & \\
\hline
\end{tabular}

Tabla 2. Parámetros estructurales de la zona tectónica. SEG segmento estructural. LAT, latitud referencial. LS, longitud estructural de la falla. IE, inmersión estructural. Mw, momento de magnitud. DF, distancia focal. T, tasa de desplazamiento de segmentos.

\section{Sismicidad contemporánea \\ histórica}

Desde 1541 hasta el 2008, el catálogo de sismicidad histórica del Ecuador registra 128 terremotos (Io=MM, desde VI hasta XI) y 987 observaciones de efectos físicos de daños en edificaciones y en el terreno (Egred, 1975, 2002). El catálogo describe los parámetros de: (1) distancia de hipocentros inferior a $40 \mathrm{Km}$, (2) intervalo de magnitudes comprendida entre $5.9 \leq \mathrm{Mw} \leq 8.8$, e (3) intensidad macrosísmica comprendida entre $\mathrm{VIII} \leq \mathrm{MM} \leq \mathrm{XI}, \quad \mathrm{MM}=$ escala macrosísmica de Mercalli.

Para la complementación de este catálogo, las informaciones sobre localidad de epicentros, intensidad máxima estimada, máxima magnitud asociada, distancia de área epicentral y efectos cosísmicos en el terreno, han sido obtenidas del análisis y de la combinación de varios catálogos, tales como: (a) "National Earthquake Information Center" -NEIC, (b) Centro Regional de Sismología para América del Sur" - CERECIS, e (c) "Instituto Geofísico de la Escuela Politécnica Nacional IGEPN, (ver en bibliografía ReNaSis).

Las crónicas de Teodoro Wolf (1872) también proporcionan información sobre los datos epicentrales de sismos para Ecuador. Para estos eventos de época pre-instrumentales, en el sitio oficial de CERESIS (www.ceresis.org) están también disponibles datos de magnitudes estimadas a partir de la intensidad macrosísmica (Me). El catálogo NEIC registra terremotos instrumentales que han afectado a Ecuador desde 1973 (disponible in escala di Magnitud momento, Mw). A nivel local, la red sísmica del IG-EPN inicia a recopilar información sismológica con resolución más precisa desde 1988 (ReNaSis). En este periodo solamente un terremoto cortical (superficial) destructivo ha sido registrado, el evento del 04.08.1998 (M 7.1, ver modelo de subducción norte) con epicentro en Bahía de Caráquez (Manabí). La información sismológica disponible del IGEPN comprende actualmente sismos con Ms $\geq 4$ (ReNaSis, www.igepn.edu.ec). La conversión de escalas mb, Ms a Mw ha sido aplicado desde las formulas propuestas por Caguari (2008).

En este estudio, también aplicamos la relación de escalas de Intensidad tradicionales (MM) con una nueva escala ESI-2007 (Environment Seismic Intensity Scale). La Escala ESI-2007 mide solamente los efectos considerables en el terreno causado durante y después de un terremoto (Michetti et al., 2007). La aplicación de esta escala junto a la de Mercalli o MSK permite obtener una mejor evaluación del daño en el terreno y ambiente, especialmente en áreas donde la edificación de estructuras es ausente (ie. Chunga, 2010).

La escala de intensidad macrosísmica ESI-2007 describe los efectos geológicos en el terreno causado por terremotos, tales como: (a) desplazamiento vertical de fallas geológicas, $(b)$ tsunamis o maremotos generados en el mar por fuertes terremotos, $(c)$ licuefacción de suelos, $(d)$ derrumbes de taludes [todos de corta duración desde a hasta $\mathrm{d}],(e)$ subsidencia o hundimiento del terreno por origen natural o inducción antrópica de obras civiles en lugares inadecuados, $(f)$ deslizamiento de tierras, $(g)$ erosión y sedimentación, $(h)$ grietas en el terreno por la saturación de los suelos por fuertes precipitaciones, y (i) variación de líneas costeras por los levantamientos o hundimientos tectónicos [todas de larga duración desde e hasta i]. 
Dependiendo de la ubicación geográfica y ambiente sedimentario, se describe a continuación los tipos de efectos geológicos que pueden generarse con un terremoto con grado de intensidad ESI $=$ X (referencia, Michetti et al, 2007), las definiciones son referidas como: (a) Las rupturas primarias llegan a ser primordiales. Las rupturas de tierra (fallamiento superficial) pueden extenderse por varias decenas de kilómetros de largo, con desplazamiento lateral alcanzado entre los 50 a 100 $\mathrm{cm}$ y más (hasta aprox. 1 a $2 \mathrm{~m}$ en caso de fallas inversas y 3 a 4 para fallas normales). Las fosas tectónicas y las depresiones alargadas se desarrollan; para los terremotos con hipocentros muy superficiales, tales como eventos volcanotectónicos; las longitudes de las rupturas pueden ser mucho más bajas. La subsidencia o el levantamiento tectónico de la superficie del terreno con máximos valores en el orden de pocos metros puede ocurrir. (b) Grandes deslizamientos y caídas de rocas (> 105 - $106 \mathrm{~m} 3)$ son frecuentes, prácticamente indiferentes al estado del equilibrio de las pendientes, causando lagos de barreras temporales o permanentes. Las orillas de los ríos, los terraplenes y las excavaciones artificiales típicamente colapsan. Terraplenes y represas de tierra pueden incluso incurrir en serios daños. El área afectada es generalmente hasta $5.000 \mathrm{~km} 2$. (c) Muchos manantiales cambian perceptiblemente su proporción de flujo y/o elevación del afloramiento. Algunos pueden secarse o desaparecer, generalmente temporalmente. Variaciones en el nivel del agua son observadas en los pozos. (d) La temperatura del agua a menudo cambia en manantiales y/o pozos. El agua en los lagos y los ríos llegan a ser con frecuencia fangosos. (e) Aberturas de grietas en el terreno hasta más de $1 \mathrm{~m}$ de ancho son frecuentes, principalmente en depósitos aluviales flojos y/o suelos saturados; en las rocas competentes la abertura alcanza varios decímetros. Anchas grietas desarrolladas en caminos pavimentados (asfalto o empedrados), así como ondulaciones por la presión. (f) Licuefacción, con emersiones de agua y compactación del suelo, puede cambiar el aspecto de zonas extensas (planicies); volcanes de arenas incluso de $6 \mathrm{~m}$ de diámetro o más; la subsidencia vertical es igual o > $1 \mathrm{~m}$; grandes y largas grietas debido a extensiones laterales son comunes. (g) las cámaras cársticas pueden colapsar, formando grandes sumideros por desplome. (h) Grandes deslizamientos son frecuentes bajo el nivel del mar en áreas costeras. (i) Grandes olas desarrolladas en agua tranquilas y de corrientes, y se estrellan violentamente contra las orillas. Las aguas de corrientes (ríos y canales) e inmóviles (lagos) puede desbordarse de sus cauces. Los tsunamis alcanzan las áreas costeras, con olas de marea hasta unos pocos metros de alto. (j) Los árboles se sacuden vigorosamente; ramas o incluso troncos de los árboles muy frecuentemente se rompe y caen, si el equilibrio es ya inestable. (k) En áreas secas las nubes de polvo pueden levantarse desde la tierra. (m) Piedras, incluso si están bien aferradas al suelo, pueden saltar de la tierra, dejando impresiones típicas en suelos blandos (no consolidados).

\section{Determinación de Isosistas de Intensidad}

El catálogo de sismos perceptibles (1541 - 2000) documentados de los registros de CERESIS y IGEPN no tiene suficiente datos para evaluar la peligrosidad sísmica de sectores industriales o áreas densamente pobladas (CERESIS, www.ceresis.org). Un análisis estadístico de los datos macrosísmicos recopilados y evaluados resulta muy difícil. Asimismo, los criterios valorados con respecto al catálogo de isosistas, no permiten obtener resultados confiables mediante técnicas de interpolación automatizada, pues los puntos que pudieran analizarse espacialmente no tendrían siempre el mismo peso en lo que respecta al valor de su intensidad, al tiempo que la no homogeneidad de su distribución introduce una complicación adicional y sólo permite en la mayoría de los casos una modelación general (Chuy, 2003).

Sin embargo estos datos de Intensidad pueden ser útiles para elaborar modelos de isosistas elípticas que indiquen los máximos niveles de sismicidad, es así, que recurrimos a analizar 1166 datos de sismos hipocentrales con magnitudes $5.1 \leq \mathrm{M} \leq 8.8$, entre las latitudes $2^{\circ} \mathrm{N}$ a $5^{\circ} \mathrm{S}$ y longitudes $75^{\circ} \mathrm{W}$ a $82^{\circ} \mathrm{W}$. Posteriormente, modelamos las distancias de isosistas para la región costera del Ecuador; aplicando la relación de conversión propuesta por Gere \& Shah, 1984 y la relación de conversión de magnitud (Ms) a Intensidad propuesta por Fedotov y Shumilina (1971):

$$
\mathrm{I}=1,5 \mathrm{M}-2,63 \log \mathrm{r}-0,0087 \mathrm{r}+2,5
$$

donde $r$ es la distancia hipocentral, I la intensidad y M la magnitud Ms del terremoto. Esta relación con la calidad de los parámetros determinados de los terremotos perceptibles permitió obtener un primer modelo de isosistas de máximos valores de intensidad para la región costera del Ecuador incluyendo a aquellas áreas no pobladas y de difícil acceso y que instrumentalmente registran sismos de magnitudes moderados. Los niveles de sismicidad estimados desde análisis de grados de intensidades macrosísmicas, comprenden valores entre VII hasta $\mathrm{X}$. 

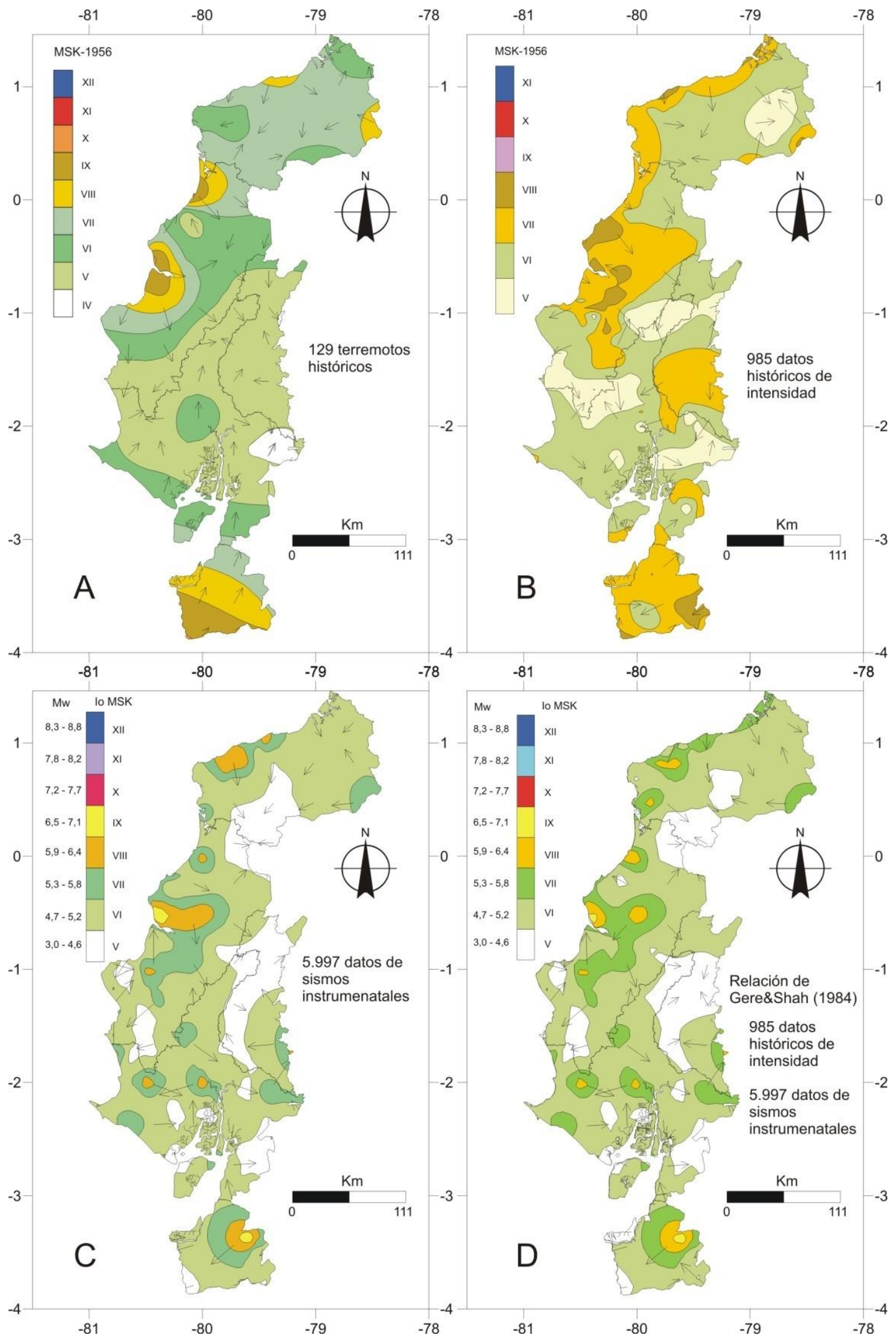

Figura 6. Niveles de sismicidad activa y modelos macrosísmicos desarrollados para el Litoral Ecuador. Los registros de terremotos aplicados a los modelos propuestos fueron obtenidos a partir de: (A), 129 terremotos históricos de intensidad MSK-1956 y ESI-2007; (B), 985 datos de intensidad a lo largo del todo el territorio nacional; (C), 5997 datos de sismos instrumentales de $M \geq 4 \leq 8,8$; y (D) integración de todos los datos indicados aplicando la relación de Gere \& Shah, 1984. 


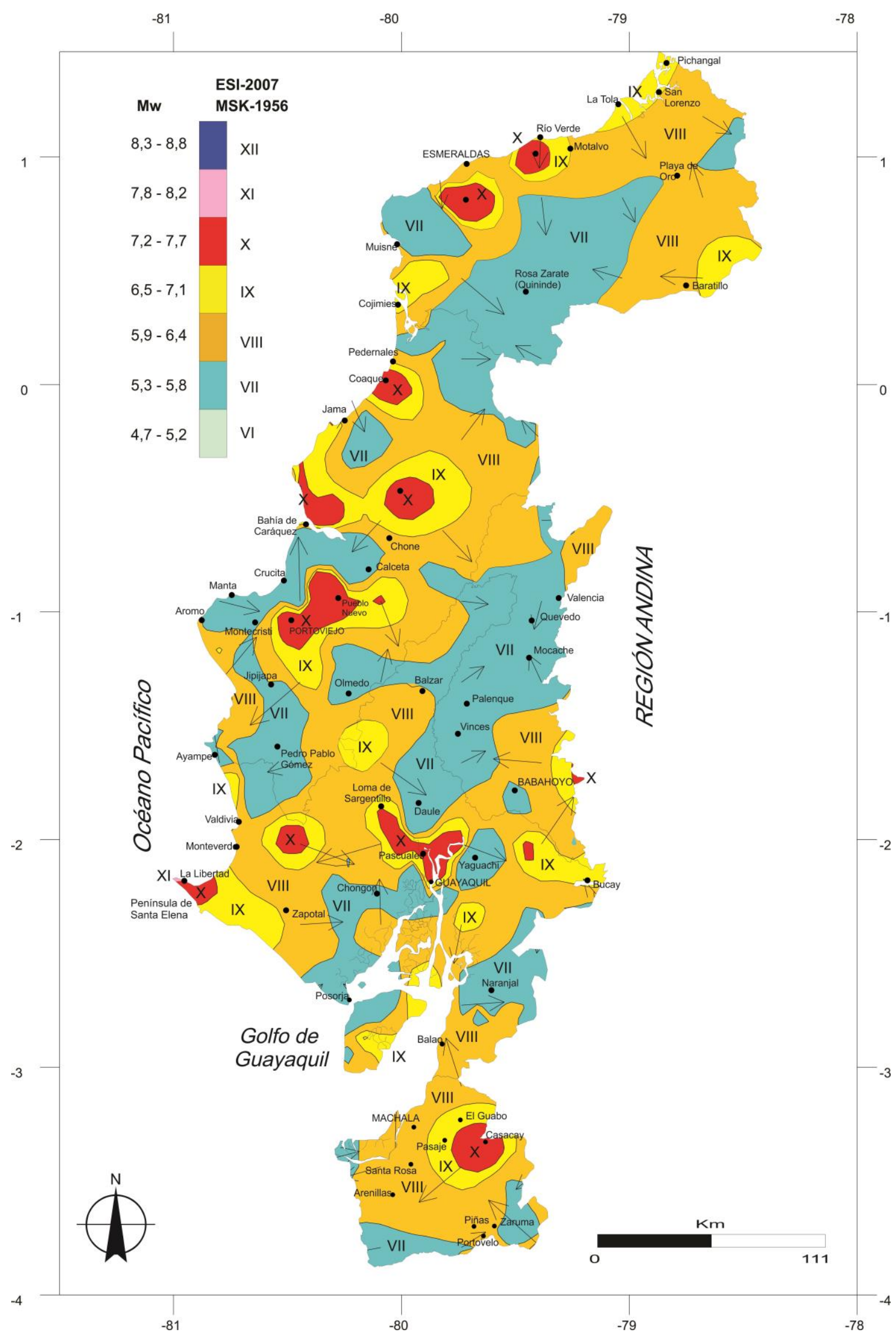

Figura 7. Estimación de máximos niveles de sismicidad para el Litoral Ecuatoriano. Escalas de intensidades esperadas MSK-1956, ESI-2007 y su relación con los momentos de magnitudes (Mw). Datos de sismos desde 1541 - 2008. Las flechas indican la dirección del gradiente del campo de intensidad. 


\section{Conclusión}

En el plano del slab, los sismos con profundidades $\geq 100 \leq 120 \mathrm{Km}$, se distribuyen cerca del litoral costero prolongándose al interior del continente siguiendo la continuidad de la zona de Benioff con proyecciones paralelas a la cordillera Andina. En el modelo de subducción sur, la distribución espacial es mucho más densa que en el modelo Norte. Sismos distribuidos a profundidades $>300 \mathrm{Km}$ son concentrados en el oriente ecuatoriano. En el litoral ecuatoriano plano de falla del slab varía muy poco en su inmersión hacia el Este, incrementando su buzamiento estructural en la región andina.

La integración de los datos de intensidad y de magnitud ha permitido determinar el máximo valor esperado durante un terremoto y la dirección del

\section{Referencia Bibliográfica}

Barazangi M., Isacks, B.L. (1976). Spatial distribution of earthquakes and subduction of the Nazca plate beneath South America: Geology, v. 4, p. 686-692.

Bourdon E., Eissen J-P. (2003). Magmatic response to early aseismic ridge subduction: the Ecuadorian margin case (South America), Earth and Planetary Science Letters, 205(3-4): 123-138.

Beck, Susan L. (1992). The May 14, 1942, Ecuador earthquake and subduction along the Colombia-Ecuador Trench. Seismological Research Letters, vol.63, no.1, pp.28.

Bellier, O., Sébrier, M., Pramumijoyo, S., Beaudouin, Th., Harjono, H., Bahar, I., Forni, O. (1997). Paleoseismicity and seismic hazard along the Great Sumatra Fault (Indonesia). J. Geodynamics Vol 24, Nos 1-4, pp.169-183.

Chiou B S.J., Youngs R.R. (2007). Chiou and Youngs PEER-NGA Empirical Ground Motion Model for the Average Horizontal Component of Peak Acceleration Pseudo-Spectral Acceleration for Spectral Periods Ranging from 0.01 to $10 \mathrm{~s}$. Interim Report for USGS review, dated June 14, 2006, revised July 10, 2006.

Chunga K., Quiñónez Ma. (2013). Evidencia sedimentaria de tsunamis en la planicie aluvial de Villamil Playas, Golfo de Guayaquil. Revista Acta Oceanográfica del Pacífico Vol. 18. In press.

Chunga K., Michetti A., Gorshkov A., Panza G.F., Soloviev A., Martillo C. (2011). Aplicación del método de zonación morfo-estructural para identificar nudos sismogénicos en la región costera y cadenas montañosas de los Andes septentrionales gradiente de campo de intensidad para el litoral ecuatoriano (ver, Figura 4). Localmente, para la ciudad de Guayaquil se estima una máxima magnitud entre 6,5 a 7,2 y una intensidad ESI-2007 de IX. En Libertad y Salinas los valores de intensidad ESI-2007 pueden alcanzar entre IX a X y la máxima magnitud entre 7,2 a 8,2. Para Portoviejo se pueden asumir los mismos valores de Guayaquil, con un considerable incremento de intensidad y magnitud al noreste del sector Pueblo Nuevo (Figura 7). Para Rio Verde, Portoviejo, Zaruma y Bahía de Caráquez se pueden asumir valores similares de Guayaquil. Una confrontación con el mapa macrosísmico de Egred (IG-EPN) y el propuesto por nosotros, permite individualizar nuevas áreas de interés con altos niveles de sismicidad.

del Ecuador. Revista Acta Oceanográfica del Pacífico Vol. 16, pág. 120 - 144.

Chunga K. (2010). Shallow crustal earthquakes and seismic zonation for Ecuador through the integration of geological, seismological and morphostructural data. University of Insubria. Ph.D. Thesis, p. 165. (Italian version).

Chunga K. (2010). Shallow crustal earthquakes and seismic zonation for Ecuador through the integration of geological, seismological and morphostructural data. University of Insubria. Ph.D. Thesis, p. 165. (Italian version).

Chunga K., León C., Quiñónez M., Benítez S., Montenegro G. (2005). Seismic Hazard Assessment for Guayaquil City (Ecuador): Insights from Quaternary Geological Data. Abstract Vol. Dark Nature - Rapid natural Change and Human Responses, Final Meeting of the Dark Nature Project, Como, Italy, p. 55-56.

Chunga K., Zamudio Y., Egred J., Marín G., Quiñónez M., Iturralde D. (2005). The 12 Dic, 1953, Earthquake, Ms 7.3, Ecuador-Peru Border Region: A case study for applying the New INQUA Intensity Scale Abstract Vol. Dark Nature - Rapid natural Change and Human Responses, Final Meeting of the Dark Nature Project, Como, Italy, p. 57-58. Dark Nature Project.

CERESIS (1985). Catálogo de terremotos para América del Sur. Datos de hipocentros e intensidades. Ecuador. www.cerecis.org

Dumont, J.F., Santana, E., Vilema, W., Pedoja, K., Ordonez, M., Cruz, M., Jimenez, N., and Zambrano, I., 2005, Morphological and microtectonic analysis of Quaternary deformation 
from Puna and Santa Clara Islands, Gulf of Guayaquil, Ecuador (South America): Tectonophysics, v. 399, p. 331-350.

Egred, J. (1975). Breve historia de los principales terremotos ecuatorianos. Revista Geofísica (México, D.F.), no.3, pp.21-36.

Egred, J. (2008). Historia sísmica del Ecuador. Aún por publicar. Información proporcionada por el Autor. Instituto Geofísico - Escuela Politécnica Nacional.

Eguez, A., Alvarado, A., Yepes, H., Machette, M.N., Costa, C.H., Dart, R.L., and Bradley, L.-A., 2003, Database and map of Quaternary faults and folds of Ecuador and its offshore regions: U.S. Geological Survey Open-File Report 03-289.

Espinoza J. (1992). Terremotos tsunamigénicos en el Ecuador. Acta Oceanográfica del Pacífico, 7(1), 21-28.

Gere, J.M., and Shah, H.C. (1984). Terra non ferma. New York: W. H. Freeman and Co., 203 p.

Gutscher, M.-A., Malavieille, J., Lallemand, S., Collot, J.-Y. (1999). Tectonic segmentation of the North Andean margin: impact of the Carnegie Ridge collision. Earth and Planetary Science Letters $168,255-270$

Mendoza, C., Dewey, J.W. (1984). Seismicity associated with the great Colombia-Ecuador earthquakes of 1942, 1958, and 1979; implications for barrier models of earthquake rupture. Bulletin of the Seismological Society of America, vol.74, no.2, pp.577-593.

Michetti, A.M.; Esposito, E.; Gürpinar, A.; Mohammadioun, B.; Mohammadioun, J.; Porfido, E.; Rogozhin, E.; Serva, L.; Tatevossian, R., Vittori, E., Audemard, F., Comerci, V., Marco, S. McCalpin, J., Mörner, N.A. (2004). The Scale INQUA: An innovative approach for assessing earthquake intensities based on seismically-induced ground effects in natural environment. Special paper. Memorie - Descrittive della Carta Geologica d'Italia, Vol. LXVII, E. Vittori and V. Comerci eds.. APAT-Dip. Difesa del Suolo/ Servizio Geologico d'Italia. Roma.

Nocquet J.M., Mothes P., Alvarado A. (2008). Geodésica, geodinámica y ciclo sísmico en Ecuador. http://renag.unice.fr/regal/PERSO/JMN/publis/geodesia_ecuador_texto_y_figuras.pdf

Pennington, W.D., 1981, Subduction of the eastern Panama Basin and seismotectonics of northwestern South America: Journal of Geophysical Research, v. 86, p. 10753-10770.

Petersen, M., Dewey, J., Hartzell, S., Mueller, C., Harmsen, S., Frankel, A., Rukstales, K. (2004). Probabilistic seismic hazard analysis for Sumatra,
Indonesia and across the Southern Malaysian Peninsula. Tectonophysics 390, 141-158.

ReNaSis. Red Nacional de Sismografos del Instituto Geofisico, Escuela Politenica Nacional de Quito. http://www.epn.edu.ec/

Silgado, F.E. (1957). El movimiento sísmico del 12 de diciembre de 1953. Boletín de la Sociedad Geológica del Perú, vol.32, Part 2, pp.225-238.

Spikings, R. A., Winkler, W., Seward, D., Handler, R. (2001). Along-strike variations in the thermal and tectonic response of tehe continental Ecuadorian Andes to the collision with heterogeneous oceanic crust. Earth and Planetary Science Letters 186, 57-73.

Swenson, J.L.; Beck, S.L. (1996). Historical 1942 Ecuador and 1942 Peru subduction earthquakes, and earthquake cycles along Colombia-Ecuador and Peru subduction segments. PAGEOPH, Vol.146, no.1, pp.67-101.

Tassara A., Yanez G. (2003). Relación entre el espesor elástico de la litosfera y la segmentación tectónica del margen andino $\left(15-47^{\circ} \mathrm{S}\right)$. Rev. geol. Chile, vol.30, no.2, p.159-186. ISSN 0716-0208.

Vannucchi P., Galeotti, S., Clift, P.D., Ranero, C.R., and von Huene, R., 2004, Long-term subduction erosion along the Guatemala margin of the Middle America trench: Geology, v. 32, p. $617-$ 620.

Vargas C., Duran J.P. (2005). State of strain and stress in northwestern of South America. Earth Sci. Res J. Vol 9, No. 1: 43-50.

Wells, D. L. and Coppersmith, K. J., 1994, New empirical relationships among magnitude, rupture length, rupture width, rupture area, and surface displacement: Bulletin of the Seismological Society of America, v. 84, p. 974-1002.

Winter, T.; Avouac, J.P.; Lavenu, A. (1993). Late Quaternary kinematics of the Pallatanga strike-slip fault (central Ecuador) from topographic measurements of displaced morphological features. Geophysical Journal International, vol.115, no.3, pp.905-920.

Witt, C., Bourgois, J., François, M., Ordoñez, M., Jiménez, N., and Sosson, M., 2005, Development of the Golfo de Guayaquil (Ecuador) as an effect of the North Andean Block tectonic escape since the lower Pleistocene, 6th Internation Symposium on Andean Geodynamics, Volume Extended Abstracts: Barcelona, Spain, p. 804-808.

Wolf (1872). Terremotos y temblores. Apéndice al Capítulo VII. Revista de los terremotos principales desde el tiempo de la conquista. Geografía y Geología del ecuador, p. 658p. 